\title{
TRIBOLOGICAL INVESTIGATION OF PLA-BASED NANOCOMPOSITES BY SCRATCH AND WEAR EXPERIMENTS
}

\author{
TODOR BATAKLIEV* \\ OLEM, Institute of Mechanics, Bulgarian Academy of Sciences, \\ Acad. G. Bonchev St., Bl. 4, Sofia 1113, Bulgaria
}

[Received: 23 September 2019. Accepted: 03 February 2020]

doi: 10.7546/JTAM.50.20.02.01

\begin{abstract}
Nanocomposites of multiwall carbon nanotubes (MWCNTs) and graphene nanoplatelets (GNPs) incorporated in polylactic acid were studied. Characterization and understanding of tribological behavior requires the exact definition of the scratch and wear conditions and look on the morphological features of the composite materials. The materials have been prepared by melt extrusion technique as a first step of the process. The received pellets having nanofiller loading up to $12 \mathrm{wt} \%$ were used to produce filaments suitable for 3D-printing. Scratch tests were performed on extremely smooth surface of 3D-printed samples applying constant normal force of $2 \mathrm{~N}$ with micro-cutting diamond blade. The scratch resistance of $12 \mathrm{wt} \%$ GNP/PLA nanocomposite was found to be twice higher than the neat PLA used as reference.
\end{abstract}

KEY WORDS: nanocomposites, carbon nanofillers, polylactic acid, mechanical properties, scanning electron microscopy.

\section{INTRODUCTION}

In recent years, the polylactic acid (PLA), an ecologically friendly polymer material, is being widely used in scientific research as a matrix for nanocomposites because of its biodegradable properties [1-5]. However, PLA possesses relatively low glass transition temperature, low thermal dimensional stability, and mechanical ductility, a fact limiting the scale of its applications. A significant number of researchers have dealt with the insertion of carbon nanofillers to improve the mechanical features of PLA [6-8]. The addition of graphene to thermoplastic polymers has been reported to modify the composites making them to exhibit superior mechanical strength while retaining their flexibility and rendering increment of wear resistance and reduction of friction due to the graphene two-dimensional arrangement of $\mathrm{sp}^{2}$ bonded carbon atoms [9-11]. It should be noticed that there is insufficient published literature concerning the scratch resistance of PLA-based nanocomposites although their promising tribological properties. Scratch resistance represents an important property in many applications [12-15]. The wear properties of PLA and PLA-graphene

\footnotetext{
${ }^{*}$ Corresponding author e-mail: todorbat@gmail.com
} 
composites have been evaluated by ball-on-disk wear tests [16]. A two-stage coefficient of friction $(C O F)$ behavior has been observed for PLA-graphene. Initially, PLA-graphene exhibited a $65 \%$ decrease in $C O F$ as compared to PLA. During the second stage, PLA-graphene approached similar $C O F$ behavior and value of PLA $(\sim 0.58)$. Concerning the grapheme, containing composite it can be seen dependence of $C O F$ data on the applied normal force unlike the pure PLA where the shape of the curves is similar at any presented load. The correlation between coefficient of friction $(C O F)$ and scratch depth has been explored over $\mathrm{ZnO} / \mathrm{PMMA}$ composites with various amount of reinforcement [17]. It was observed that the friction coefficient went down with reducing the scratch depth and increasing the amount of $\mathrm{ZnO}$ in the composites. Both scratch depth and $C O F$ values of the composites were found to decrease compared to the neat PMMA. Frictional sliding experiments using conical diamond indenter and consisting of normal indentation, transient regime of frictional sliding and steady state frictional sliding have been performed to calculate the overall resistance to penetration as well as the overall friction coefficient [18]. The tests were conducted in order to evaluate the effect of plastic strain characteristics on the scratch response under different surface friction conditions. Several articles have addressed the improvement of Young's modulus and tensile strength in PLA-based nanocomposites modified with carbon nanotubes or graphene [19-21]. However, there is a lack in the literature of focusing on the tribological behavior of GNP/PLA and MWCNT/PLA composites. Novelty of that research is the use of completely flat 3D-printed nanocomposite samples appropriate for macromechanical testing.

\section{EXPERIMENTAL}

Ingeo ${ }^{\mathrm{TM}}$ Biopolymer PLA-3D850 with MFR 7-9 $\mathrm{g} / 10 \mathrm{~min}\left(210^{\circ} \mathrm{C}, 2.16 \mathrm{~kg}\right)$ was purchased from Nature Works. TNIGNP-Industrial Graphene nanoplatelets - average thickness $<30 \mathrm{~nm}$ and diameter of $\sim 5-7 \mu \mathrm{m}$; purity $90 \%$ and TNIMH4OH functionalized MWCNTs (TNIMH): OD: 10-30 nm; Length: 10-30 $\mu \mathrm{m}$; SSA $=110 \mathrm{~m}^{2} / \mathrm{g} ; \mathrm{EC}>100 \mathrm{~S} / \mathrm{cm}$; purity $95 \%$ were supplied from TimesNano, China.

Monofiller (GNP/PLA and MWCNT/PLA) and bifiller (GNP/MWCNT/PLA) composites were made by means of melt extrusion technique. Strips with geometry $60 \times 12 \times 2.4 \mathrm{~mm}$, printing density of $100 \%$ and possessing very low roughness were prepared by 3D-printing using filament with diameter of $1.75 \mathrm{~mm}$ and applying FDM technique combined with layer-to-layer deposition. The set of samples was made with German RepRap X-400 Pro 3D-printer having two printing heads and equipped with Simplify 3D-slicer.

Scratch and wear experiments were carried out on CETR-UMT-2 tribological testing module system (Bruker, USA). The 3D-printed nanocomposite samples were scratched by force control method applying normal load of $2 \mathrm{~N}$ and using micro- 
cutting diamond blade with tip radius of $0.4 \mathrm{~mm}$. Wear behavior of monofiller and bifiller composite samples was studied through reciprocating motion test made with chrome stainless steel ball (6.35 mm diameter) and enforcing friction load of $2 \mathrm{~N}$.

SEM analysis of the nanocomposites was made by using a field emission scanning electron microscope (FESEM, mod. LEO 1525, Carl Zeiss SMT AG, Oberkochen, Germany). 3D-printed samples having scratches were immersed in liquid nitrogen, then cut to make them appropriate to the holder and finally coated with gold (layer thickness $250 \AA$ ) using a sputter coater (mod. B7341, Agar Scientific, Stansted, UK).

\section{Results AND Discussion}

\subsection{SCRATCH EXPERIMENTS}

Two sets of 3D-printed composite samples having single filler loading were analyzed by applying scratch test. The behavior of the coefficient of friction at $2 \mathrm{~N}$ constant normal force as a function of the scratch length was monitored during the tests. The coefficient of friction values were measured with the aid of Viewer Software.

The addition of GNPs contributed to significantly improved tangential force $\left(F_{x}\right)$ and accordingly to higher $C O F$ and scratch resistance of the PLA-based nanocomposites at the relevant applied normal force of $2 \mathrm{~N}$ [13], see Fig. 1. This is due to the creation of network structure formed by the filler suppressing the moving of the diamond blade through the sample. Near 100\% registered COF improvement in the graphene curve compared to pure PLA showed more pronounced effect of the

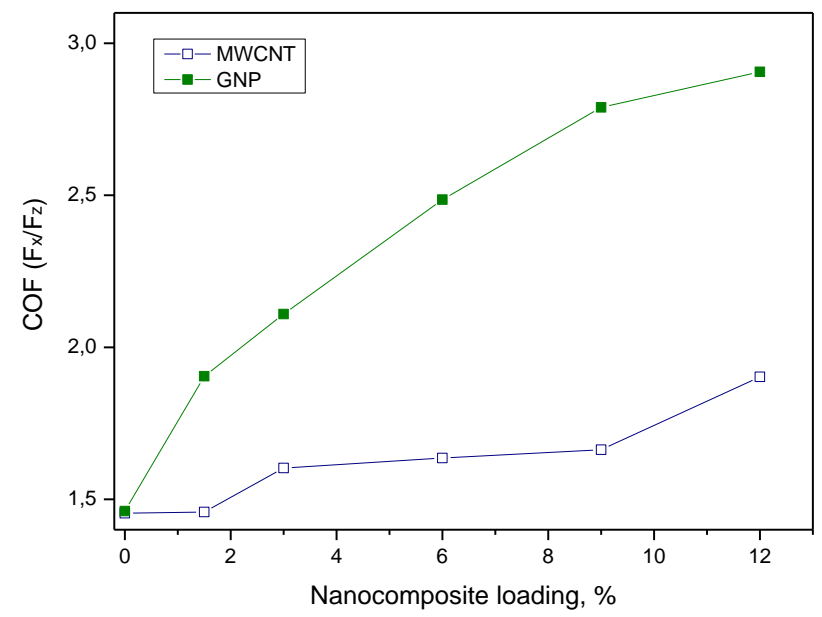

Fig. 1: $C O F$ dependence on carbon monofiller content recorded from scratch test data. 
new composite structure in the material scratch behavior of GNP/PLA samples. The slope of the curve presenting the received $C O F$ values of composite incorporated with carbon nanotubes is more flat comparing to graphene composites curve but the scratch resistance of the samples is rising as well with filler loading increment. That finding could be explained with distinct mechanism and magnitude of load transfer between the polymer die and the carbon nanotubes reflecting in different mechanical properties of the nanocomposite. The investigation of friction coefficient in singlepass scratch test revealed that the induced tangential force $F_{x}$ increases by reinforcing the samples with higher carbon filler loading. It can be assumed that the interfacial friction coefficient $\mu_{s}$ is more considerable regarding the nanocomposites with highest graphene or carbon nanotubes content. Moreover, the overall friction coefficient $C O F=F_{x} / F_{z}$ at scratch tends to the interfacial friction coefficient when the apex angle of the blade $\alpha$ tends to $180^{\circ}$ [22]

\subsection{WEAR EXPERIMENTS}

The wear behavior of carbon monofiller nanocomposite samples compared to neat polylactic acid is depicted in Fig. 2. The 3D-printed strips were set up to reciprocating friction test on linear motion drive under dry sliding conditions and enforcing $2 \mathrm{~N}$ normal constant load. All wear tests were conducted for time period of $300 \mathrm{~s}$ each one of them. The coefficient of friction parameters in the graphics represent average values corresponding to the whole test time period.

Pure PLA exhibited a higher $C O F$ in the range of $0.15-0.16$ whereas the GNP

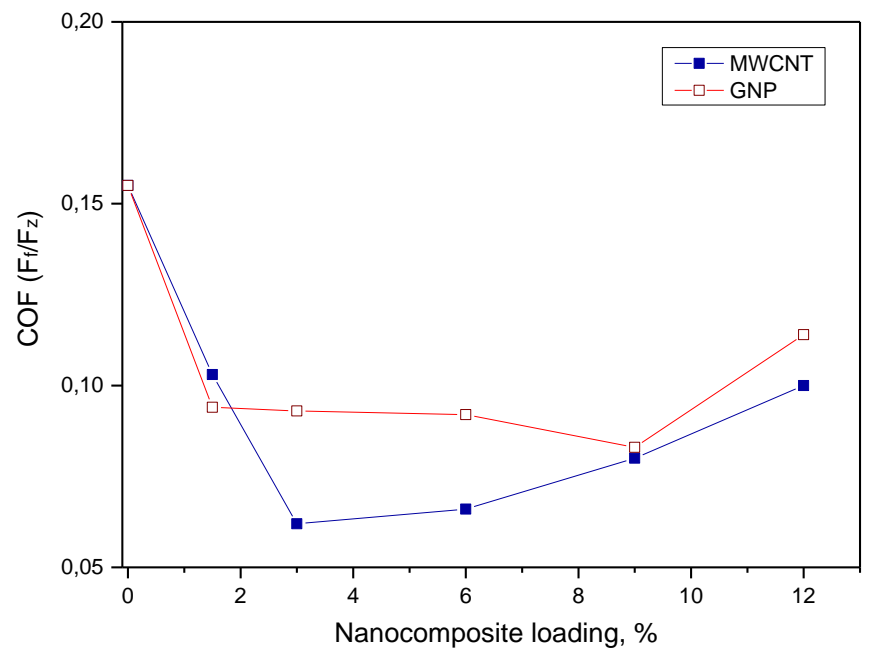

Fig. 2: $C O F$ dependence on carbon monofiller content recorded from wear test data. 


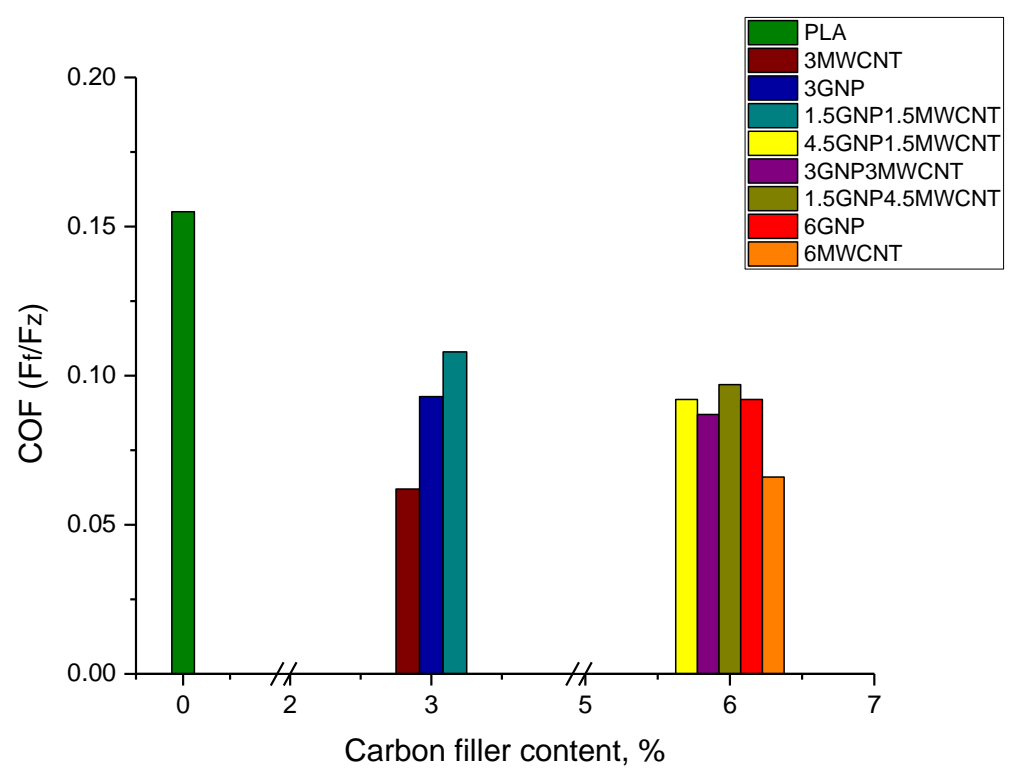

Fig. 3: $C O F$ dependence on $3 \mathrm{wt} \%$ and $6 \mathrm{wt} \%$ carbon monofiller and bifiller content recorded from wear test data.

containing composite samples showed lower $C O F$ values around 0.10 with 0.11 maximum for the compound possessing the highest graphene loading of $12 \mathrm{wt} \%$. A two-stage coefficient of friction $(C O F)$ behavior can be observed for the carbon nanotubes reinforced PLA composites. Initially, up to $3 \mathrm{wt} \%$ MWCNT loading there is serious decrease in $C O F$ reaching value of 0.07 as compared to the neat PLA. During the second stage, it can be seen increment in $C O F$ up to 0.10 for the composite $12 \mathrm{wt} \% \mathrm{MWCNT/PLA}$. This approach is similar to $C O F$ behavior of the PLA-based composite with $12 \mathrm{wt} \%$ graphene loading. The exerted normal stress during wear test could lead to dissociation of carbon flakes from the nanocomposite matrix resulting in significantly lower coefficients of friction compared to pure PLA [16]. To understand the contributing factor of using two carbon nanofillers in PLA matrix on the wear resistance of the materials a series of nine bifiller composite samples was examined and juxtaposed to the monofiller samples using the same experimental conditions, see Figs. 3 and 4. Minor negative effect of using both GNPs and MWCNTs in composite die can be mentioned about 1.5GNP1.5MWCNT/PLA sample having higher $C O F$ than the monofiller samples with same carbon filler content (Fig. 3). The presence of GNPs in nanocomposite structure appeared to be a precondition to receive a bit higher $C O F$ along wear experiment compared to the monofiller sam- 


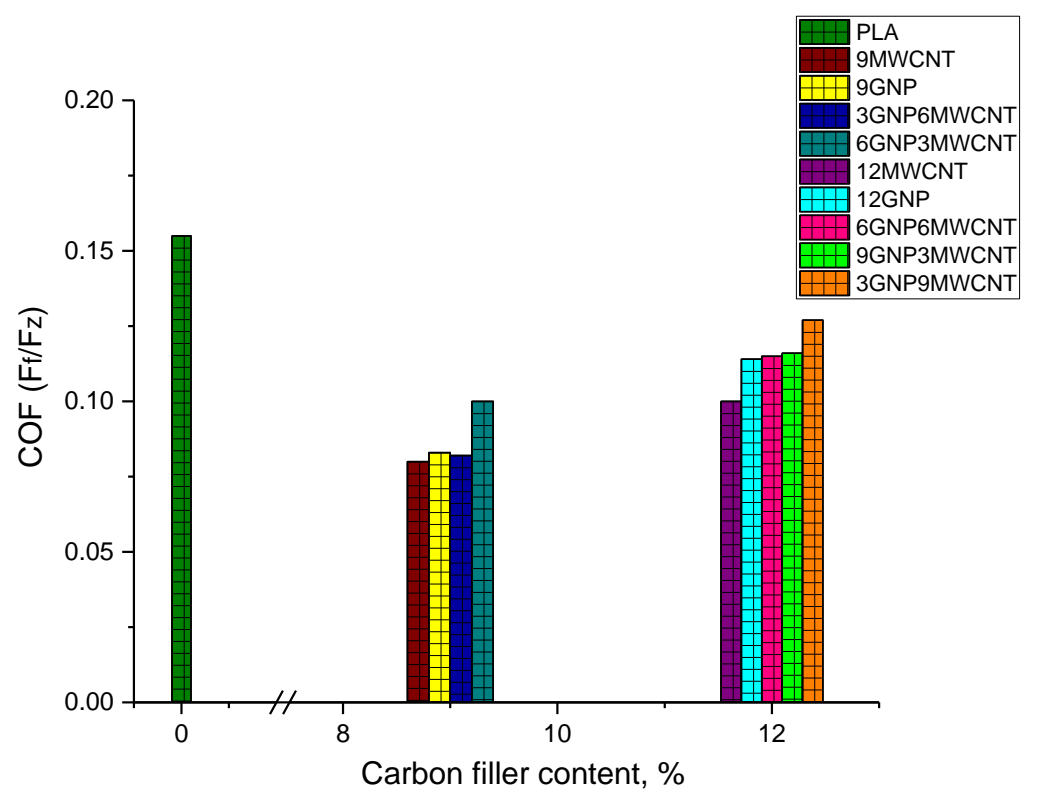

Fig. 4: $C O F$ dependence on $9 \mathrm{wt} \%$ and $12 \mathrm{wt} \%$ carbon monofiller and bifiller content recorded from wear test data.

ples reinforced with MWCNTs as it can be seen in Figs. 3 and 4. This aftermath is assigned to more considerable self-lubricating effect of the multiwall carbon nanotubes incorporated in composite PLA matrix influencing the respond of material to superficial friction.

Insignificant decrease in wear properties as result of mixing up graphene and carbon nanotubes was noticed by the received $C O F$ values of 6GNP3MWCNT/PLA and 3GNP9MWCNT/PLA as well (Fig. 4). However, the obtained data disclosed reduction in coefficient of friction for all tested nanocomposite samples meaning decline in the friction force compared to pure PLA. This finding could be related to the improved wear resistance as result of graphene and carbon nanotubes mechanical strength along the planar direction and easier shear capability among the composite layers.

\subsection{SEM ANALYSIS}

The morphology of the scratches on the surface of 3D-printed monofiller composite strips is shown in Figs. 5a and 5b. The scratch behavior of the monofilled $6 \mathrm{wt} \%$ GNP/PLA and 6 wt $\%$ MWCNT/PLA nanocomposites could be explained with the worn tracks profile presented in Fig. 5. The higher tangential force $\left(F_{x}\right)$ received for 


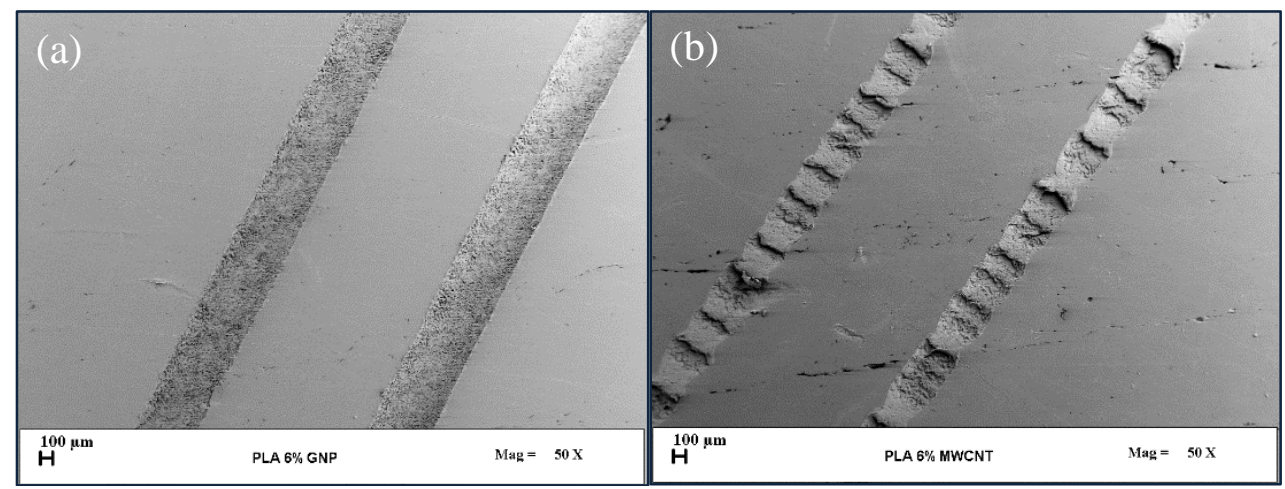

Fig. 5: Top surface microstructure of scratches on 3D-printed nanocomposite samples.

the graphene composite and leading to higher coefficient of friction might be due to the more uniform and smooth chevron notches made by the diamond blade. Moreover, in the micrographs it can be seen that the structure of the composite modified with carbon nanotubes is much more porous and full of cracks resulting in lower $C O F$ received from scratch test compared to the $C O F$ of GNP/PLA composite.

\section{CONCLUSIONS}

GNP/PLA and MWCNT/PLA composites with various amount of reinforcement have been synthesized by melt blending method. The coefficient of friction $(C O F)$ from scratch and wear experiments of the 3D- printed PLA-based nanocomposites was determined. The SEM images revealed two types of failure mechanisms over the worn tracks depending on the carbon nanofiller in the composite die. The nanocomposites showed higher tangential force during scratch test comparing to neat PLA. Dry sliding reciprocating wear tests disclosed lower values of $C O F$ for the monofiller and bifiller compounds regarding the unreinforced polylactic acid. A possible reason for this could be the self-lubricating effect of GNP/PLA and MWCNT/PLA composites. The promising results in the present study designate the feasibility of 3D-printed GNP/PLA and MWCNT/PLA nanocomposites for potential use in many industrial applications.

\section{ACKNOWLEDGMENTS}

This work was supported by the European Regional Development Fund within the OP "Science and Education for Smart Growth 2014-2020", Project CoE "National center for/of mechatronics and clean technologies", No BG05M2OP001-1.001-0008-C01. 
The author is grateful for the funding from the European Union's Horizon 2020MSCA-RISE-734164 Graphene 3D-Project and for the support by the H2020-FETGraphene Flagship-881603 Graphene Core 3.

The author would like to acknowledge the contribution of the COST Action CA15107 and Bilateral collaboration between IMech, BAS and CNR, Napoli/Portici (20192021).

\section{REFERENCES}

[1] B. Fiedler, F.H. Gojny, M.H.G. Wichmann, M.C.M. Nolte, K. Schulte (2006) Fundamental aspects of nano-reinforced composites. Composites Science and Technology 66 3115-3125.

[2] J.H. Chang, Y.U. AN, G.S. SuR (2003) Poly(lactic acid) nanocomposites with various organoclays. I. Thermomechanical properties, morphology, and gas permeability. Journal of Polymer Science Part B 41 94-103.

[3] A.M. Pinto, M. Susana, I.C. Goncalves, F.M. Gama, A.M. Mendes, F.D. MAGALHES (2013) Biocompatibility of poly(lactic acid) with incorporated graphenebased materials. Colloids and Surfaces B. 104 229-238.

[4] Y. Du, T. Wu, N. Yan, M.T. Kortschot, R. Farnood (2014) Fabrication and characterization of fully biodegradable natural fiber-Reinforced poly (lactic acid) composites. Composites Part B: Engineering 56 717-723.

[5] X. Li, Y. Xiao, A. Bergeret, M. Longerey, J. Che (2014) Preparation of Polylactide/Graphene Composites from Liquid-Phase Exfoliated Graphite Sheets. Polymer Composites 35 396-403.

[6] J.N. Coleman, V. Barron, K. Hedicke, W.J. Blau (2002) Morphological and mechanical properties of carbon-nanotube-reinforced semicrystalline and amorphous polymer composites. Applied Physics Letters 81 5123-5125.

[7] S.C. TJONG (2006) Structural and mechanical properties of polymer nanocomposites. Materials Science and Engineering: R. 53 73-197.

[8] E. Bafekrpour, M. Salehi, E. Sonbolestan, B. Fox (2014) Effects of microstructural parameters on mechanical properties of carbon nanotube polymer nanocomposites. Journal of Sciences, Islamic Republic of Iran 21 403-413.

[9] X.H. Lin, J.G. GAI (2016) Synthesis and applications of large-area single-layer graphene. RSC Advances 6 17818-17844.

[10] V. Singh, D. Joung, L. Zhai, S. Das, S.I. Khondaker, S. Seal (2011) Graphene based materials: Past, present and future. Progress in Materials Science 56 1178-1271.

[11] H.S. DongN, S.J. QI (2015) Realizing the potential of graphene-based materials for biosurfaces - A future perspective. Biosurface and Biotribology 1 229-248.

[12] F. Wredenberg, P.L. LARSSON (2009) Scratch testing of metals and polymers: Experiments and numerics. Wear 266 76-83. 
[13] H. Porwal, M. Kasiarova, P. Tatarko, S. Grasso, J. Dusza, M.J. Reece (2015) Scratch behaviour of graphene alumina nanocomposites. Advances in Applied Ceramics 114 34-41.

[14] M. Olek, K. Kempa, S. Jurga, M. Giersig (2005) Nanomechanical properties of silica-coated multiwall carbon nanotubes poly(methyl methacrylate) composites. Langmuir 21 3146-3152.

[15] I. Campos-Silva, A.D. Contla-Pacheco, A. Ruiz-Rios, J. MartnezTrinidad, G. RodrgueZ-CAstro, A. MEneses-Amador, W.D. Wong-AngeL (2018) Effects of scratch tests on the adhesive and cohesive properties of borided Inconel 718 superalloy. Surface and Coatings Technology 349 917-927.

[16] J. Bustillos, D. Montero, P. Nautiyal, A. Loganathan, B. Boesl, A. AGARWAL (2018) Integration of Graphene in Poly(Lactic) Acid by 3D-Printing to Develop Creep and Wear-Resistant Hierarchical Nanocomposites. Polymer Composites 39 3877-3888.

[17] H. Chakraborty, A. Sinha, N. Mukherjee, D. Ray, P.P. ChattopadHYAY (2013) A study on nanoindentation and tribological behavior of multifunctional ZnO/PMMA nanocomposite. Materials Letters 93 137-140.

[18] S.C. Bellemare, M. DaO, S. Suresh (2008) Effects of mechanical properties and surface friction on elasto-plastic sliding contact. Mechanics of Materials 40 206-219.

[19] S.I. Moon, F. Jin, C. Lee, S. Tsutsumi, S.H. Hyon (2005) Novel carbon nanotube/poly(L-lactic acid) nanocomposites; their modulus, thermal stability, and electrical conductivity. Macromolecular Symposia 224 287-295.

[20] A.M. Pinto, C. Goncalves, I.C. Goncalves, F.D. Magalhaes (2016) Effect of biodegradation on thermo-mechanical properties and biocompatibility of poly(lactic acid)/graphene nanoplatelets composites. European Polymer Journal 85 431-444.

[21] M.S.Z.M. DESA, A. HASSAN, A. ARSAD (2013) The effect of natural rubber toughening on mechanical properties of poly(lactic acid)/multiwalled carbon nanotube nanocomposite. Advanced Materials Research 747 639-642.

[22] G. SUbHASH, W. ZHANG (2002) Investigation of the overall friction coefficient in single-pass scratch test. Wear 252 123-134. 